American Journal of Applied Sciences 9 (5): 752-758, 2012

ISSN 1546-9239

(C) 2012 Science Publications

\title{
Genetic Diversity of Some Saudi Barley (Hordeum Vulgare L.) Landraces Based on Two Types of Molecular Markers
}

\author{
${ }^{1,2}$ Mohamed Abdel Hamid Mohamed El-Awady, \\ ${ }^{1,2}$ Adel Ahmed El-Sayed El-Tarras and ${ }^{2}$ Salah El-Din El-Assal \\ ${ }^{1}$ Biotechnology and Genetic Engineering Research Unit, \\ Scientific Research Center, Faculty of Medicine, Taif University, KSA \\ ${ }^{2}$ Department of Genetics, Faculty of Agriculture, Cairo University, Egypt
}

\begin{abstract}
Problem statement: Very little is known about the genetic diversity and morphological variability present in barley landrace in KSA, a country experiencing loss of biodiversity because of replacement of landraces with modern landraces. Approach: The molecular markers RAPD and ISSR were used as an efficient tools to estimate the intra-and inter-cultivar polymorphism among six barley KSA landraces collected from different geographical regions in order to assess the genetic relationships and develop cultivar-specific molecular fingerprints. The long term objective was to use these fingerprints to identify molecular markers that co-segregate and could be used in isolating gene(s) which controlling some important traits, thereafter could be used in breeding programs (marker assisted selection). Results: Out of 20 and 10 primers of RAPD and ISSR, respectively, a clear and reproducible band profile of 13 RAPD primers and 7 ISSR primers were obtained. In RAPD analyses, 61 out of 111 bands $(54.6 \%)$ were polymorphic. The number of alleles ranged from 5-15 per primer, with an average of 8.54 per primer. In ISSR analyses, a total of 53 alleles were detected, among which 16 alleles $(30.2 \%)$ were polymorphic. The number of alleles per primer ranged from 5-10 with an average of 7.57 alleles per ISSR primer. The mean Polymorphism Information Content (PIC) values were 0.45 and 0.37 for RAPD and ISSR markers, respectively. Conclusion: ISSR is better than RAPD to detect genetic diversity among the barley landraces. The RAPD's and ISSR's have confirmed each other and the ISSR results are more realistic comparing to RAPD results regarding to the geographical distribution of the six barley landraces. The outcome of this investigation can help strengthen the exiting pool of information on barley that may help assess national barley programs in KSA.
\end{abstract}

Key words: Barley, Random Amplified Polymorphic DNA (RAPD), Inter-Simple Sequence Repeat (ISSR), Polymorphism Information Content (PIC), Kingdom of Saudi Arabia (KSA)

\section{INTRODUCTION}

Barley was first cultivated 10,500 years ago in the Fertile Crescent and is one of the founder crops of Eurasian agriculture (Saisho and Purugganan, 2007). In some regions of the world, especially the sub-regions, barley landraces still cultivated. Barley landraces are considered as the evolutionary link between wild barley (Hordeum spontaneum K. Koch) and modern barley landraces. Barley landraces are genetically heterogeneous populations comprising inbreeding lines and hybrid segregates generated by a low level of random out crossing in each generation (Shewry, 1992). Moreover, having evolved across thousands of years in a multitude of environments and local farming systems, these landraces have developed abundant patterns of variation and would represent a largely untapped reservoir of useful genes for adaptation to biotic and abiotic stresses (Brush, 1995). Assessment of the extent of genetic variability within barley, including the wild relatives, is fundamental for barley breeding and the conservation of genetic resources and is particularly useful as a general guide in the choice of parents for breeding hybrids (Hou et al., 2005).

In the Kingdom of Saudi Arabia (KSA), less than 2 percent of the land is used for cultivation. Crops are grown mainly in the southwest of the kingdom, where there is rainfall sufficient for farming, or in areas where oases provide enough ground water for irrigation. Landraces of important crops such as barley and wheat

Corresponding Author: Mohamed El-Awady, Biotechnology and Genetic Engineering Research Unit, Scientific Research Center, Faculty of Medicine, Taif University, KSA 
have persisted in situ because of the nature of the farming systems. By growing a mixture of diversified local materials, farmers were always able to select varieties adapted to local environmental conditions. Since the 1990s, many modern landraces of barley were introduced to the kingdom to replace the local landraces resulted in the gradually decrease of these variable source of biodiversity. However, barley landraces can still be found in isolated and marginal areas, such as mountains and oases where traditional landraces are still grown. Very little is known about the genetic diversity present in these barley landrace.

Molecular markers have been proved to be valuable tools in the characterization and evaluation of genetic diversity within and between species and populations. It has been shown that different markers might reveal different classes of variation (Russell et al., 1997). It is correlated with the genome fraction surveyed by each kind of marker, their distribution throughout the genome and the extent of the DNA target which is analyzed by each specific assay (Davila et al., 1999a; 1999b). The advent of the Polymerase Chain Reaction (PCR) favored the development of different molecular techniques such as Random Amplified of Polymorphic DNA (RAPD), Simple Sequence Repeats (SSR or microsatellite), Sequence Tagged Sites (STS), Random Amplified Microsatellite Polymorphism (RAMP) and Inter-Simple Sequence Repeat (ISSR) and so on (Nagaoka and Ogihara, 1997). These molecular markers had been used in barley for detecting genetic diversity, genotype identification, genetic mapping (Tanyolac, 2003). Of these techniques, RAPD has several advantages, such as simplicity of use, low cost and the use of small amount of plant material. RAPDs were proved to be useful as genetic markers in the case of self-pollinating species with a relatively low level of intra specific polymorphism, such as hexaploid wheat (Joshi and Nguyen, 1993) and cultivated barley (Tinker et al., 1993). ISSR markers, which involve PCR amplifications of DNA using a primer composed of a microsatellite sequence anchored at 3' or 5' end by 2-4 arbitrary, could be used to assess genetic diversity (Qian et al., 2001). ISSRs have been used for cultivar identification in maize (Pejic et al., 1998), potatoes (Prevost and Wilkinson, 1999), wheat (Nagaoka and Ogihara, 1997), bean (Metais et al., 2000), Diplotaxis (Martín and Sanchez-Yelamo, 2000) and barley (Hou et al., 2005).

The major objectives of this work were focusing on estimating of polymorphism among six barley KSA landraces, Hail; Qassem; Taif; Gizan; Bahah and Asser, using RAPD and ISSR molecular markers, as efficient tools in order to assess the genetic relationships and develop genotype-specific molecular fingerprints to be used in barley breeding programs.

\section{MATERIALS AND METHODS}

Materials: Six barley landraces samples were collected from different locations of KSA and named according to the collection area as Hail; Qassem; Taif; Gizan; Bahah and Asser.

Methods: Isolation and purification of genomic DNA from the different barley landraces.

Fresh young healthy barley leaves were collected from the studied barley landraces and grounded to powder with liquid $\mathrm{N}_{2}$ using a mortar and pestle. Genomic DNA was isolated from leaf samples using the procedure described by the plant isolation kit (Jena Bioscience, Germany).

\section{RAPD analysis:}

PCR reaction and condition: A total of 20 random primers were used to detect the polymorphism. The sequence of the 13 primers that produce a clear scoriable and reproducible banding pattern is shown in (Table 1). The amplification performed in a $25 \mu \mathrm{l}$ reaction volume containing about $3 \mu \mathrm{l}\left(10 \mathrm{ng} \mu \mathrm{L}^{-1}\right)$ genomic DNA, $3 \mu \mathrm{L}$ primer (Operon Technologies Inc.) and $12.5 \mu \mathrm{L}$ master mix (Promega) and $6.5 \mu \mathrm{L}$ of PCR water. The PCR temperature profile was applied through Bio-Rad C1000 thermal cycler (Germany) that was programmed with an initial step of $5 \mathrm{~min}$ at $94^{\circ} \mathrm{C}$; the amplification reaction was carried out using 40 cycles of $60 \mathrm{~s}$ at $94^{\circ} \mathrm{C}$, an annealing step of $1 \mathrm{~min}$ at $37^{\circ} \mathrm{C}$ and an elongation step of $1 \mathrm{~min}$ at $72^{\circ} \mathrm{C}$; and finally a 7 min extension at $72^{\circ} \mathrm{C}$. The amplification products were resolved by electrophoresis in a $1,5 \%$ agarose gel containing ethidium bromide $\left(0.5 \mu \mathrm{g} \mathrm{mL} \mathrm{L}^{-1}\right)$ in $1 \mathrm{X}$ TBE buffer at 95 volts. PCR products were visualized on UV light and photographed using a gel documentation system (Bio-Rad® Gel Doc-2000).

ISSR analysis: Ten ISSR primers were tested using a specific and optimal annealing temperature for each one (Table 2). PCR reactions were performed in a volume of $20 \mu \mathrm{l}$ Bio-Rad C1000 thermal cycler (Germany). The reaction mixture contained $0.6 \mu \mathrm{L}$ of each primer, 100 $\mu \mathrm{M}$ of each deoxinucleotide, 0.5 units of Go Taq polymerase (Promega), 10×Taq buffer containing 2.5 $\mathrm{mM} \mathrm{MgCl} 2$ and $10 \mathrm{ng}$ of template DNA. Amplification reaction was $94^{\circ} \mathrm{C} / 5 \mathrm{~min}$, followed by 30 cycles of $94^{\circ} \mathrm{C} / 1 \mathrm{~min}, 4-60^{\circ} \mathrm{C}$ (specific for each primer) $/ 1 \mathrm{~min}$ and $72^{\circ} \mathrm{C} / 2$ min and ending with an extension step of $7^{\circ} \mathrm{C} / 7 \mathrm{~min}$. PCR products were analyzed using agarose $(2 \% \mathrm{w} / \mathrm{v})$ electrophoresis gels stained with ethidium bromide and only bands with high intensity and well separated were selected. 
Am. J. Applied Sci., 9 (5): 752-758, 2012

Table 1: List of random primers that have been used for RAPD analysis with their sequence, polymorphism $\%$ and Polymorphic information content value (PIC) for each marker

\begin{tabular}{lllrlrl}
\hline $\begin{array}{l}\text { Prime } \\
\text { No }\end{array}$ & $\begin{array}{l}\text { Primer } \\
\text { name }\end{array}$ & $\begin{array}{l}\text { Primer } \\
\text { sequence }\end{array}$ & $\begin{array}{l}\text { Number of } \\
\text { total bands }\end{array}$ & $\begin{array}{l}\text { Amplified } \\
\text { fragments (bp) }\end{array}$ & $\begin{array}{c}\text { Polymorphism } \\
(\%)\end{array}$ & PIC \\
\hline 1 & OP-A06 & GGTCCCTGAC & 5.0 & $200-1700$ & 20.0 & 0.32 \\
2 & OP-A07 & GAAACGGGTG & 9.0 & $500-2000$ & 25.0 & 0.38 \\
3 & OP-A08 & GTGAGGTAGG & 9.0 & $600-1500$ & 33.3 & 0.45 \\
4 & OP-A10 & GTGATCTCC & 7.0 & $350-1600$ & 43.0 & 0.50 \\
5 & OP-B01 & TGATCCCTG & 8.0 & $400-3000$ & 100.0 & 0.90 \\
6 & OP-B02 & GGACTGGAGT & 6.0 & $500-2500$ & 50.0 & 0.50 \\
7 & OP-B03 & TGCGCCTTC & 5.0 & $400-1600$ & 40.0 & 0.48 \\
8 & OP-B04 & TGCGCCCTTC & 6.0 & $700-2000$ & 17.0 & 0.28 \\
9 & OP-B05 & CTGCTGGGAC & 11.0 & $400-2000$ & 64.0 & 0.46 \\
10 & OP-B10 & TTCGAGCCAG & 11.0 & $200-1500$ & 42.0 & 0.49 \\
11 & OP-C01 & GATGAGCCAG & 6.0 & $100-2000$ & 50.0 & 0.50 \\
12 & OP-C02 & GATGACCGCC & 7.0 & $600-1500$ & 70.0 & 0.42 \\
13 & OP-C03 & TGGACCGGTG & 13.0 & $300-1700$ & 93.0 & 0.13 \\
Average & & & 7.8 & & 49.8 & 0.45 \\
\hline
\end{tabular}

Table 2: List of Primers that have been used for ISSR analysis with their sequence, Amplified Fragments (bp), Number of total bands, Polymorphism \% and PIC value for each marker

\begin{tabular}{llcccc}
\multicolumn{7}{c}{ bands, Polymorphism $\%$ and PlC value for each marker } \\
\hline $\begin{array}{l}\text { Prime } \\
\text { Name }\end{array}$ & $\begin{array}{l}\text { prime } \\
\text { total bands }\end{array}$ & $\begin{array}{c}\text { Amplified } \\
\text { fragments } \\
\text { sp) }\end{array}$ & $\begin{array}{c}\text { Polymorphism } \\
(\%)\end{array}$ & PIC \\
\hline 1-UBC80 & ATATATATATATATATT & - & - & - & - \\
2-UBC807 & AGAGAGAGAGAGAGAGT & 5.0 & $500-2000$ & - & - \\
3-UBC828 & TGTGTGGTGGATGTGGAT & 6.0 & $400-1700$ & 33.30 & 0.41 \\
4-UBC878 & CATGGTGTTGGATGGAT & 6.0 & $400-1000$ & 33.30 & 0.45 \\
5-UBC899 & CATGGTGGTCGATTGTTCA & - & - & - & - \\
6-UBC822 & TCTCTCTCTCTCTCTCA & 8.0 & $500-1800$ & 25 & 0.47 \\
7-UBC814 & CTCTCTCTCTCTCTCTA & - & - & - & - \\
8-UBC835 & AGAGAGAGAAGAGAGAYT & 10.0 & $800-1000$ & 30.00 & 0.42 \\
9-UBC840 & GAGAGAGAGAGAGAYT & 10.0 & $100-2000$ & 30.00 & 0.42 \\
10-UBC847 CACACACACACACACARC & 6.0 & $300-500$ & 33.30 & 0.45 \\
Average & & 7.4 & & 25.80 & 0.37 \\
\hline
\end{tabular}

Data scoring and statistical analysis: To ensure the absence of artifacts, bands were carefully selected from replicated amplifications (three times). Amplified bands designated by their primer code and their size in base pairs. Data recorded as discrete variables: 1 for the presence and 0 for the absence of a similar band. Only intense and reproducible bands appearing on the gel were scored. Band scoring was analyzed using Gene Tools-gel analysis software of SPSS ver. 16. The Polymorphic Information Content value (PIC) refers to the value of a marker for detecting polymorphism within a population and depends on the number of detectable alleles and the distribution of their frequency. PIC was calculated using the equation:

$$
P I C i=1-\sum \begin{gathered}
n \\
\text { Pij2 } \\
j=1
\end{gathered}
$$

where, PICi is the polymorphic information content of a marker $\mathrm{i}$; Pij is the frequency of the jth pattern for marker $\mathrm{i}$ and the summation extends over $\mathrm{n}$ patterns (Anderson et al., 1993).

\section{RESULTS}

RAPD-PCR fingerprinting of barley landraces: The use of RAPD's molecular markers was aiming to show fast and reliable discrimination of any variations. The DNA of the six landraces of KSA barley was used as templates for 20 RAPD primers.

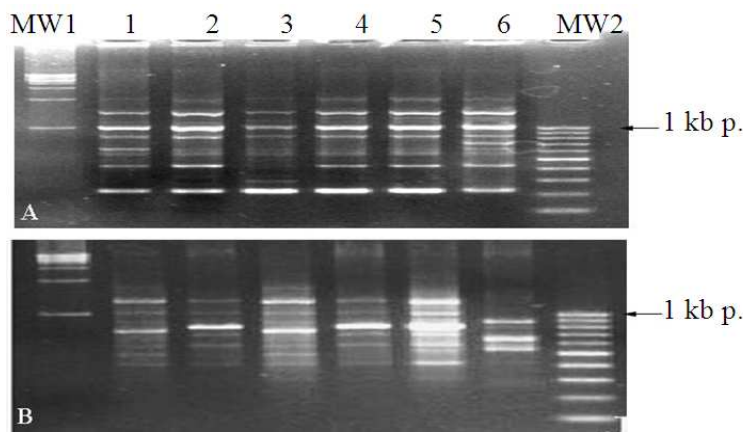

Fig. 1:RAPD fingerprinting of $6 \mathrm{KSA}$ barley landraces (Hordium vulgare L.). MW1: is $1 \mathrm{~kb}$. ladder and MW1 is 100 bp. ladder. (1) Taif; (2) Hail; (3) Qassem; (4) Asser; (5) Bahah; (6) Gizan. (A) Primer OP-B10; and (B) Primer OP-C08

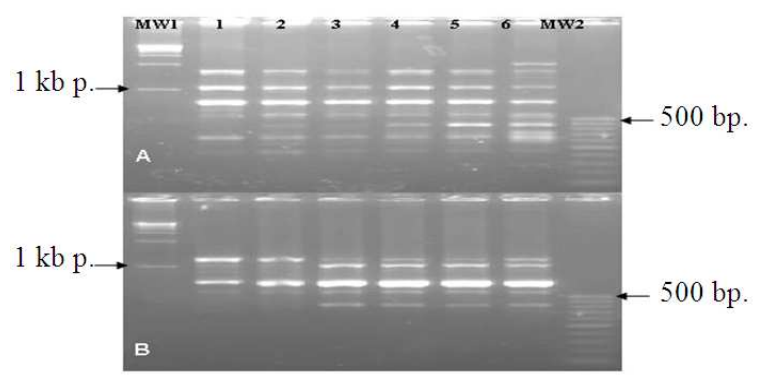

Fig. 2: ISSR fingerprinting of $6 \mathrm{KSA}$ barley landraces (Hordium vulgare L.). MW1: is $1 \mathrm{~kb}$. ladder and MW1 is $50 \mathrm{bp}$. ladders. (1) Taif; (2) Hail; (3) Qassem; (4) Asser; (5) Bahah; (6) Gizan. (A) Primer UBC840 and (B) Primer UBC847

Only 13 primers produced a clear, scoreable banding pattern. Number of bands obtained with each primer, polymorphism \% and PIC value of each primer were illustrated in Table 1.

Total of 102 bands were obtained with the average of 7.8 bands for each primer. The highest band number was produced with primer OP-C08 (15 bands) while the lowest was produced with primers OP-A06 and OPB03 (5 bands). The RAPD banding obtained with primers OP-B10 and OP-C05 are illustrated in Fig. 1. The size of the amplified fragments was ranged between 100 and 3000 bp length (Table 1). Primer OPB01 showed the highest polymorphism (100\%), while primer OP-B04 showed the lowest with only $17 \%$ (Table 1). Remarkably, primer (OP-B05) has showed specific product for Qassem and Bahah landraces, between 300 and 400 bp. length. While primers OP$\mathrm{B} 10$ has showed a specific product for Qassem landrace between 400 and 500 bp. length (Fig. 1). The primer OP-C08 has showed a specific product for Gizan cultivar, with 800 bp. length. 


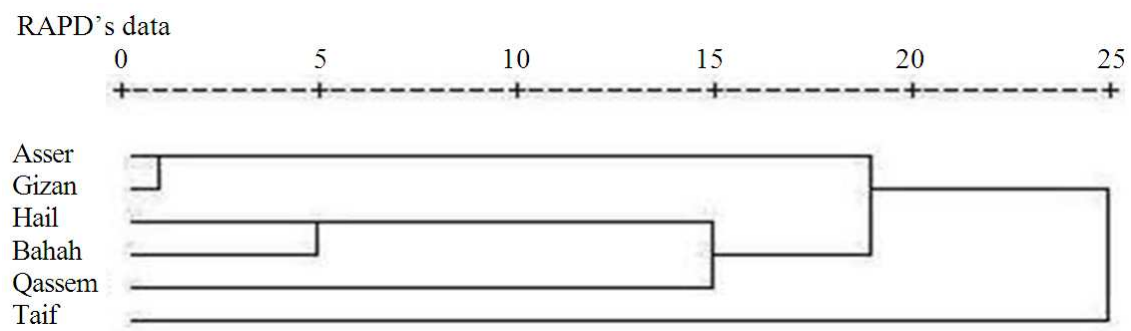

(a)

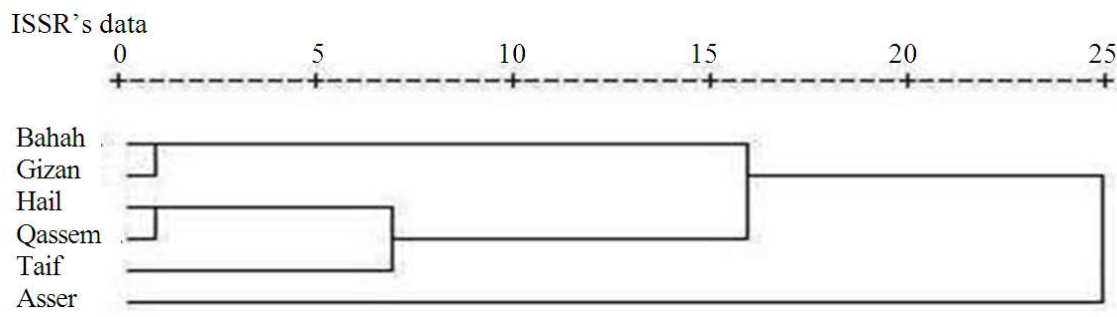

(b)

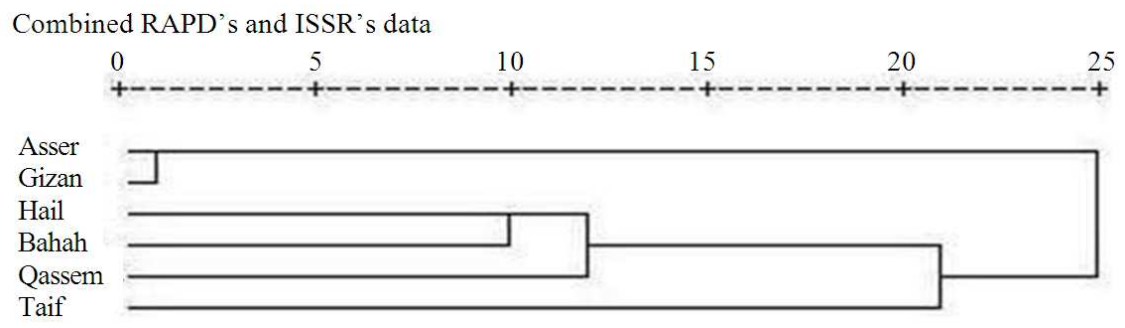

(c)

Fig. 3: Phylogenetic analysis among the six barley landraces using RAPD and ISSR. (A) the analysis of RAPD's data; (B) the analysis of ISSR's data and (C) the combined RAPD's and ISSR's data

ISSR-PCR fingerprinting of barley landraces: In this study, we have used the DNA of six KSA barley landraces, as templates for 10 ISSR primers. However, only seven primers showed clear and scorable bands. The ISSR-PCR reaction of primer (UBC807) has recorded 5 fragments that with the molecular size ranged from 500-2000 bp. All five bands were observed in all landraces and exhibited 100\% monomorphism (data not shown). The other six ISSR primers exhibited a polymorphic banding pattern.

The total of 51 bands were obtained with the average of 7.4 bands for each primer. The highest band number was produced with primers UBC835 and UBC840 (10 bands) while the lowest was produced with primers UBC807 (5 bands). The size of the amplified fragments was ranged between 300 and 2000 bp length (Table 2). The three primers UBC828, UBC878 and UBC847 showed polymorphism \% of (33.3), while primer UBC822 showed the lowest with only $25 \%$ (Table 2). The ISSR banding pattern obtained with primers UBC840 and UBC847 are depicted in Fig. 2. A specific band for Gizan landrace, with around 2000 bp. Length was produced by the primer UBC840 has showed a specific (Fig. 2-A)

Genetic relationships as revealed by RAPD and ISSR markers: The RAPD's product showed a genetic similarity ranged from 59.6-84.4\% (Table 3). The highest genetic similarity revealed by the RAPD's analysis was $84.4 \%$ between landraces Asser and Gizan and $80.9 \%$ between landraces Hail and Bahah. On the other hand, the lowest genetic similarity was $59.6 \%$ between landraces Qassem and Taif.

The ISSRs data revealed a genetic similarity ranged from 76-93.6\% (Table 3). The highest genetic similarity revealed by the ISSR analysis was $93.6 \%$ between Bahah and Gizan landraces and 93.6\% between landraces Hail and Qaseem. On the other hand, the lowest genetic similarity was $76 \%$ between Asser and Taif landraces. The ISSR data was very close to the geographical distribution of the six landraces.

The RAPD's and ISSR's combined data were close to RAPD data and showed genetic similarity ranged between 68.2 and $84.6 \%$ (Table 3). 
Am. J. Applied Sci., 9 (5): 752-758, 2012

Table 3: Genetic dissimilarity (GS) matrices computed according to Dice coefficient fromRAPD and ISSR markers of six KSA barley landraces. (Taif; Hail; Qassem; Asser; Bahah; and Gizan). (A): is the analysis of RAPD's data; (B): is the analysis of ISSR's data and (C): is the combined RAPD's and ISSR's data

\begin{tabular}{|c|c|c|c|c|c|c|}
\hline Case & Taif & Hail & Qassem & Asser & Bahah & Gizan \\
\hline \multicolumn{7}{|c|}{ A RAPD'data } \\
\hline \multicolumn{7}{|c|}{ Taif } \\
\hline Hail & 0.745 & & & & & \\
\hline Qassem & 0.596 & 0.710 & & & & \\
\hline Asswer & 0.644 & 0.750 & 0.692 & & & \\
\hline Bahah & 0.695 & 0.809 & 0.774 & 0.701 & & \\
\hline Gizan & 0.636 & 0.727 & 0.685 & 0.844 & 0.713 & \\
\hline \multicolumn{7}{|c|}{ B ISSR'data } \\
\hline \multicolumn{7}{|c|}{ Taif } \\
\hline Hail & 0.898 & & & & & \\
\hline Qassem & 0.917 & 0.936 & & & & \\
\hline Asswer & 0.760 & 0.776 & 0.830 & & & \\
\hline Bahah & 0.843 & 0.860 & 0.917 & 0.872 & & \\
\hline Gizan & 0.820 & 0.837 & 0.894 & 0.848 & 0.936 & \\
\hline \multicolumn{7}{|c|}{ C Combined RAPD's and ISSR'data } \\
\hline \multicolumn{7}{|c|}{ Taif } \\
\hline Hail & 0.796 & & & & & \\
\hline Qassem & 0.704 & 0.786 & & & & \\
\hline Asswer & 0.682 & 0.758 & 0.739 & & & \\
\hline Bahah & 0.747 & 0.826 & 0.826 & 0.757 & & \\
\hline Gizan & 0.698 & 0.764 & 0.657 & 0.846 & 0.787 & \\
\hline
\end{tabular}

The highest genetic similarity was $84.6 \%$ between Asser and Gizan, while the lowest genetic similarity was $68.2 \%$ between Taif and Asser landraces.

Cluster analysis as revealed by RAPD and ISSR markers: Although barley landraces, Hail, Qassem and Taif were collected from the same geographical region of KSA and Bahah, Asser and Gizan were collected from another geographical region, RAPD's product in Fig. 3 showed that in a dendrogram the six barley landraces cluster comprised two major sub clusters with 19\% dissimilarity. One subcluster includes (Hail, Bahah and Qassem), with genetic similarity with similarity starts from $71 \%$ until $80.9 \%$. The other subcluster includes two barley landraces (Gizan and Asser), with similarity of $84.4 \%$. While the sixth cultivar, Taif, is falling outside of both of two subclusters (Fig. 3). This means that the environmental and biotic conditions are very powerful in causing genetic variations among barley landraces. Moreover, the classification of these varieties might be was based on morphological traits which is not so powerful comparing with the molecular markers.

ISSR results in (Fig. 3) showed that in a dendrogram the six landraces cluster comprised two subclusters with almost $16 \%$ dissimilarity. One subcluster includes (Hail, Taif and Qassem), with genetic similarity starts from $89.8 \%$ until $93.6 \%$. The other subcluster includes two barley landraces (Gizan and Bahah), with similarity of $93.6 \%$. While the sixth landrace Asser is falling outside of both of two subclusters. The ISSR results confirmed the geographical distribution, when the Hail, Taif and Qassem falled together in one group, also the other three landraces, Gizan, Asser and Taif recorded high similarity, but some speciation pressures could cause the genetic differences among the three landraces. Although the ISSR data is logically close to the nature distribution of the studied landraces, the combined RAPD and ISSR results was relatively close to the RAPD data (Fig. 3).

\section{DISCUSSION}

It is obvious that the varieties landraces in different ecological conditions of KSA have attained different genetic profiles during the time. Most important criteria for the selection of marker are: Amount of available information, simple process or work, less expenses, high rate of doing work. In attention to the fact that RAPD marker don't need the use of radioactive elements and having sample sequence in plant genome such as possibility of the use of general primers those can be applied in every species of plant therefore RAPD marker is the suitable marker for the study of genetic variations and relativity rate and also it has the ability to assess the reservoirs of inheritance of the plant species.

Due to its worldwide distribution, the assessment of the genetic diversity among barley germplasm from different countries was performed (Fernandez et al., 2002; Feng et al., 2003). In fact, it has been found that the average genetic diversity based on RAPD analysis of 18 barley accessions from Netherlands, France, Great Britain, Germany and Italy was 0.521 (Russell et al., 1997). In other conducted work on 30 barley American landraces (Pillen et al., 2000), the average genetic diversity was 0.682 . Whereas, the genetic diversity between tea populations (Kaundun and Park, 2002) was 0.18.

In the present study, the average genetic dissimilarity of barley landraces was 0.42 . These results suggest that the selected barley landraces have narrow genetic diversity, despite the high PIC value of primers used. Correlation between RAPD markers and the geographic origin is low: In deed Bahah landrace from the south of KSA was closely related to Hail and Qassem landraces which is from north regions of KSA. The landraces Taif and Qassem, collected from the same region, showed an important Genetic Diversity (GD) of $0.59 \%$. However, Hail and Qassem belonging to the same bioclimatic stage showed a low GD. This study corroborate with those found in Korean tea 
populations (Kaundun and Park, 2002). This relatively poor relationship observed between RAPD markers and the geographic origin of KSA barley landraces in this study may be explained by the neutrality of molecular markers compared with adaptive traits under harsh conditions. This study revealed the efficacy of RAPD markers in detecting the polymorphism among barley landraces and establishing relationship among them. It demonstrated the potential efficiency of molecular markers in landraces classification and indicated the feasibility of a comprehensive effort to determine the relationships among barley landraces using molecular markers. Further collection, evaluation and utilization of local germplasm, is clearly a high priority in barley improvement.

In this study, it was obvious that the dendrogram based on RAPD markers was not in accord with the dendrogram based on ISSR markers. The dendrogram generated by the ISSR matrix agrees better with the geographic origins of the genotypes than the dendrogram generated by the RAPD results. For example, three landraces Hail, Qssem and Taif (originating from north KSA), while the other three landraces, Bahah, Gizan and Asser (from south KSA) were closely clustered in the dendrogram generated by the ISSR matrix and agrees better with the geographical distribution, comparing with the RAPD dendrogram (Fig. 1a and b). Moreover, (GS) matrices computed according to Dice coefficient from ISSR markers of the six KSA barley landraces were high between those landraces that collected from the same geographical region of KSA (Table 3).

\section{CONCLUSION}

In this study local six barley landraces (Hail; Qassem; Taif; Gizan; Bahah and Asser) were collected from two different regions of KSA. Although RAPD's reactions have showed high average of polymorphism (49.8\%) comparing with ISSR markers polymorphic average $(25.8 \%)$, the ISSR results were trustable, repeatable even for intra-specific purposes and could reflect real genetic relationships among barley landraces and correlated with the geographical distribution of them. They are potentially useful markers to identify barley landraces and might be used for cultivar identification. In the crop plants such as barley, the polymorphism rate according to other molecular markers was low. The existence of polymorphic markers was an excellent choice in order to use in different breeding aims.

\section{REFERENCES}

Anderson, J.A., G.A. Churchill, J.E. Autrique, S.D. Tanksley and M.E. Sorrells, 1993. Optimization parental selection for genetic linkage maps. Genome, 36: 181-186. DOI: 10.1139/g93-024
Brush, S.B., 1995. In situ conservation of landraces in center of crop diversity. Crop Sci., 35: 346-354.

Davila, J.A., Y. Loarce and E. Ferrer, 1999a. Molecular characterization and genetic mapping of random amplified microsatellite polymorphism in barley. Theror. Applied Genet., 98: 265-273. DOI: 10.1007/s001220051067

Davila, J.A., Y. Loarce, L. Ramsay, R. Waugh and E. Ferrer, 1999b. Comparison of RAMP and SSR markers for the study of wild barley genetic diversity. Heredities, 131: 5-13. DOI: 10.1111/j.1601-5223.1999.00005.X

Feng, Z.Y., Y.Z. Zhang, L.L. Zhang and H.Q. Ling, 2003. Genetic diversity and geographical differentiation of Hordeum vulgare ssp. Spontaneum in Tibet using microsatellite markers. High Tech. Lett., 10: 46-53.

Fernandez, M., A. Figueiras and C. Benito, 2002. The use of ISSR and RAPD markers for detecting DNA polymorphism, genotype identification and genetic diversity among barley cultivars with known origin. Theror. Applied Genet., 104: 845-851. DOI: 10.1007/s00122-001-0848-2

Hou, Y.C., Z.H. Yan, Y.M Wei and Y.L. Zheng, 2005. Genetic diversity in barley from west China based on RAPD and ISSR analysis. Barley Genet. News 35: 9-22.

Joshi, C.P. and H.T. Nguyen, 1993. RAPD (random amplified polymorphic DNA) analysis based intervarietal genetic relationships among hexaploid wheats. Plant Sci., 93: 95-103. DOI: 10.1016/01689452(93)90038-2

Kaundun, S.S. and Y.G. Park, 2002. Genetic structure of six Korean Tea populations as revealed by RAPD-PCR markers. Crop. Sci., 42: 594-601.

Martín, J.P. and M.D. Sanchez-Yelamo 2000. Genetic relationships among species of the genus Diplotaxis (Brassicaceae) using inter-simple sequence repeat markers. Theror. Applied Genet., 101: 1234-1241. DOI: 10.1007/s001220051602

Metais, I., C. Aubry, B. Hamon and R. Jalouzot, 2000. Description and analysis of genetic diversity between commercial bean lines (Phaseolus vulgaris L.). Theror. Applied Genet., 101: 1207-1214. DOI: $10.1007 / \mathrm{s} 001220051599$

Nagaoka, T. and Y. Ogihara, 1997. Applicability of inter-simple sequence repeat polymorphisms in wheat for use as DNA markers in comparison to RFLP and RAPD markers. Theror. Applied Genet., 94: 597-602. DOI: 10.1007/s001220050456 
Pejic, I., P. Ajmone-Marsan, M. Morgante, V. Kozumplick and P. Castiglioni et al., 1998. Comparative analysis of genetic similarity among maize inbred lines detected by RFLPs, RAPDs, SSRs and AFLPs. Theror. Applied Genet., 97: 1248-1255. DOI: 10.1007/s001220051017

Pillen, K., A. Binder, B. Kreuzkam, L. Ramsay, R. Waugh and J. Forster et al., 2000. Mapping new EMBL-derived barley microsatellites and their use in differentiating German barley cultivars. Theror. Applied Genet., 101: 652-660. DOI: $10.1007 / \mathrm{s} 001220051527$

Prevost, A. and M.J. Wikinson, 1999. A new system of comparing PCR primers applied to ISSR fingerprinting of potato cultivars. Theror. Applied Genet., 98: 107-112. DOI: 10.1007/s001220051046

Qian, W., S. Ge and D.Y. Houng, 2001. Genetic variation within and among populations of a wild rice Oryza granulata from China detected by RAPD and ISSR markers. Theror. Applied Genet., 102: 440-449. DOI: 10.1007/s001220051665

Russell, J.R., J.D. Fuller, M. Macaulay, B.G. Hatz and A. Jahoor et al., 1997. Direct comparison of levels of genetic variation among barley accessions detected by RFLPs, AFLPs, SSRs and RAPDs. Theror. Applied Genet., 95: 714-722. DOI: 10.1007/s001220050617
Saisho, D. and M.D. Purugganan, 2007. Molecular phylogeography of domesticated barley traces expansion of agriculture in the Old World. Genet., 177: 1765-1776. DOI: 10.1534/genetics.107.079491

Shewry, P.R., 1992. Barley: Genetics, Biochemistry, Molecular Biology and Biotechnology. 1st Edn., C.A.B. International, Wallingford, ISBN-10: 0851987257, pp: 610 .

Tanyolac, B., 2003. Inter-simple sequence repeat (ISSR) and RAPD variation among wild barley (Hordeum. vulgare subsp. spontaneum) populations from west Turkey. Genet. Resour. Crop Evol., 50: 611-614. DOI: 10.1023/A:1024412814757

Tinker, N.A., M.G. Fortin and D.E. Mather, 1993. Random amplified polymorphic DNA and pedigree relationships in spring barley. Theror. Applied Genet., 85: 976-984. DOI: 10.1007/BF00215037 